\title{
The Italian registry of cystic echinococcosis (RIEC): the first prospective registry with a European future
}

F Tamarozzi (f_tamarozzi@yahoo.com) ${ }^{1}$, P Rossi ${ }^{2}$, F Galati ${ }^{3}$, M Mariconti ${ }^{4}$, G J Nicoletti ${ }^{1}$, F Rinaldi ${ }^{1}$, A Casulli $^{2}$, E Pozio ${ }^{2}$, E Brunetti ${ }^{1,4}$

1. Department of Clinical, Surgical, Diagnostic and Paediatric Sciences, University of Pavia, WHO Collaborating Centre for Clinical Management of Cystic Echinococcosis, Pavia, Italy

2. Department of Infectious, Parasitic and Immunomediated Diseases, Istituto Superiore di Sanità, Rome, Italy

3. SIDBAE, Information Technology, Istituto Superiore di Sanità, Rome, Italy

4. Division of Infectious and Tropical Diseases, San Matteo Hospital Foundation, University of Pavia, WHO Collaborating Centre for Clinical Management of Cystic Echinococcosis, Pavia, Italy

Tamarozzi F, Rossi P, Galati F, Mariconti M, Nicoletti GJ, Rinaldi F, Casulli A, Pozio E, Brunetti E. The Italian registry of cystic echinococcosis (RIEC): the first prospective registry with a European future. Euro Surveill. 2015;20(18):pii=21115. Available online: http://www.eurosurveillance.org/ViewArticle. aspx?Articleld $=21115$

Cystic echinococcosis (CE), a worldwide zoonosis, is highly endemic in southern and eastern Europe. Its actual prevalence is unknown due to the lack of efficient reporting systems designed to take into account the particular features of the disease. Neglect of CE makes diagnosis and clinical management difficult outside referral centres, with inconsistencies in clinical practice and often unnecessary procedures carried out that have associated risks and costs. The Italian registry of CE (RIEC) is a prospective multicentre registry of CE patients seen from January 2012 in Italian health centres; data are voluntarily submitted to the registry. Its aims are to show the prevalence of CE in Italy, bring the importance of this infection to the attention of health authorities, encourage public health policies towards its control, and stimulate biological, epidemiological and clinical research on CE. From January 2012 to February 2014, a total 346 patients were enrolled in 11 centres, outnumbering national reports of many CE-endemic European countries. We discuss preliminary data and challenges of the RIEC, template for the European registry of $\mathrm{CE}$, which has been implemented within the Seventh Framework Programme project HERACLES (Human cystic Echinococcosis ReseArch in CentraL and Eastern Societies) since September 2014.

\section{Background}

Cystic echinococcosis (CE) is a zoonotic disease affecting an estimated 1.2 million people worldwide, with 3 million disability-adjusted life years (DALYs) lost globally every year, although these figures are likely to be underestimates [1]. Echinococcus granulosus, the cestode causing the infection, completes its life cycle between dogs and other canids (definitive hosts) and livestock, especially sheep (intermediate hosts), with humans as accidental intermediate hosts. In humans, the parasite develops in its metacestode stage, forming cysts in organs and tissues, mainly in the liver. CE is mostly endemic in rural areas where sheep raising is practiced, such as central Asia and China, South America and Mediterranean countries [2]. In 2012 a joint FAO/World Health Organization (WHO) expert group classified E. granulosus second among the top eight ranked food-borne parasites of global public health importance [3]. Nevertheless, compared with other diseases of similar burden, CE has received much less attention and funding [4].

Human CE has been described as chronic, complex and neglected [5]. The lack of efficient reporting systems designed to take into account the particular features of this disease results in under-reporting and/or misreporting of CE. In a vicious circle, under-reporting leads to the perception that CE is not an important health problem, which in turn makes measurement of disease burden and impact on public health even more difficult. Neglect hampers the collection of good-quality data to inform control programmes and evidence-based diagnostic and therapeutic strategies. This ultimately leads to at least suboptimal clinical management of cases and allocation of public resources for treatment and control.

\section{Reasons for neglect}

Neglect of CE is due to several factors [1]: firstly, the life cycle of E. granulosus is difficult to interrupt in the absence of sustained, expensive and well-coordinated programmes. Secondly, control of the infection in humans, as opposed to livestock, does not have an impact on the global spread of infection. CE is not perceived as an important animal health problem, which is therefore not tackled. Thirdly, the burden of CE is difficult to quantify because of its geographical dispersal in vast rural areas, absence of specific symptoms, and lack of an effective disease record system. Lastly, the disease affects mostly poor pastoral communities, with 
Box

Data acquired in the Italian registry of cystic echinococcosis, January 2012-February 2014

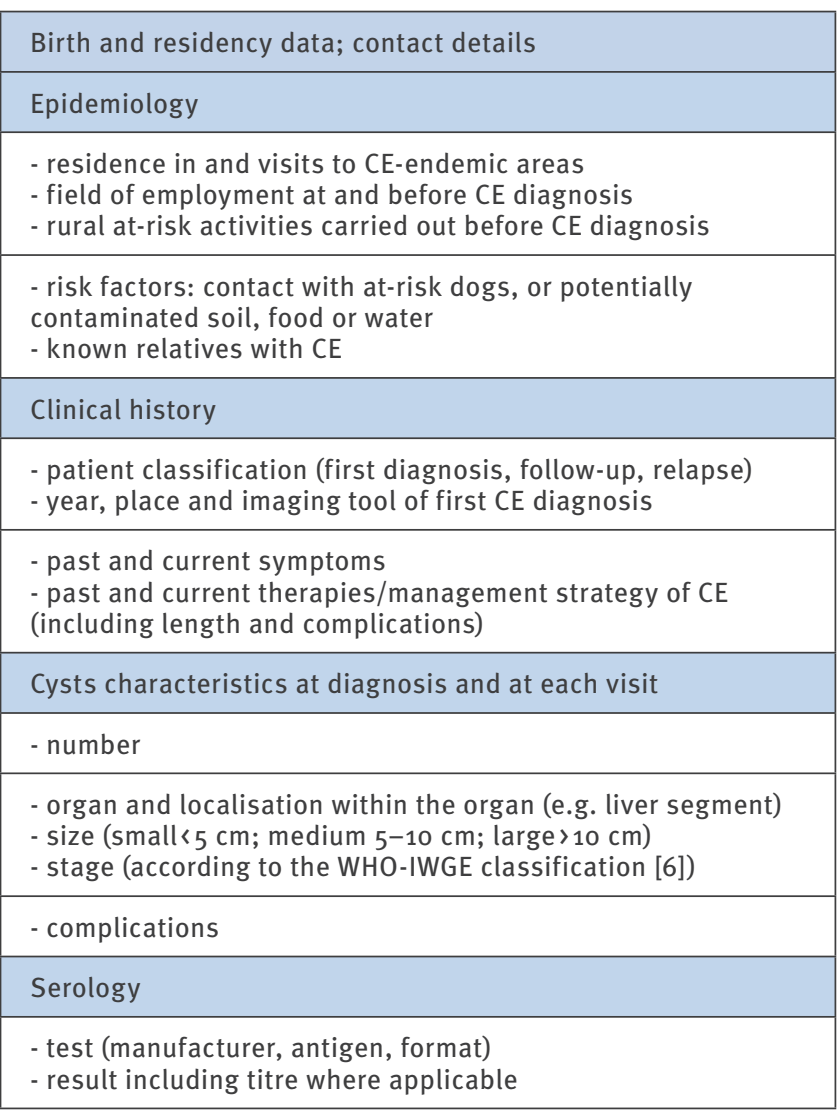

CE: cystic echinococcosis; WHO-IWGE: World Health Organization Informal Working Group on Echinococcosis.

Data are entered using fixed drop-down menus and multiplechoice options, organised in multiple data sheets (the title of each sheet is shown in bold). For all information, location, start date and end date of the event is indicated if possible. Fields for entering free text are also present where appropriate.

a low case fatality rate but with difficult and expensive diagnosis and treatment.

\section{Implications of chronicity and complexity of cystic echinococcosis}

In humans, echinococcal cysts grow slowly, passing through several stages (Figure 1), and often remain clinically silent for many years $[6,7]$. Thus, infected patients may be diagnosed long after infection and in a country different from where the infection was acquired. This compounds the difficulty in evaluating the distribution of CE. Diagnosis and clinical management of CE are complex and require a multidisciplinary approach, often available only in referral centres. Imaging, in particular ultrasound, is the mainstay of diagnosis and follow-up of abdominal CE $[6,8]$ while serology has only an ancillary role [9]. In 2003, the WHO Informal Working Group on Echinococcosis (IWGE) implemented a consensus classification of cyst stages
[6] (Figure 1). This followed the introduction of the first widely used ultrasound classification of hepatic CE proposed by Gharbi et al. [10]. The WHO-IWGE classification allows all morphological stages of cysts to be classified unequivocally, and groups them into clinical categories reflecting the current knowledge of the natural history of CE. Importantly, it provides a guide for the rational allocation of CE patients to different management options [11]. However, this consensus approach, and the use of CE classifications of any kind, are still widely underused [12], adding to the lack of prospective randomised trials evaluating the optimal stage-specific clinical management of $C E$, which hampers an evidence-based approach to this disease. Clinical management of CE still relies on expert opinion [11] and the management of the disease is often inappropriate, exposing patients (and health systems) to unnecessary treatments and costs.

\section{Reporting systems and burden of cystic echinococcosis in Italy and Europe}

CE is endemic in Italy, although its burden is difficult to estimate. Despite being a notifiable infection in animals [13], being listed among reportable occupational diseases [14] and subject to surveillance according to European legislation [15], notification of human cases ceased de facto to be compulsory in 1991. Currently, health authorities are required to provide only a yearly summary of regional data [16]; it is up to the individual physician to notify cases. As a result, no official data are transmitted to the European Centre for Disease Prevention and Control (ECDC) [17-19]. At the national level, a mean of 1,379 (SD: 442.5) yearly hospital discharges with a diagnosis of CE were recorded in 200112 , with annual incidence estimated to range from 0.18 to 6.78 per 100,000 population [20]. However, reporting based solely on hospital discharge records is inadequate, as the majority of CE cases are diagnosed and managed in an outpatient setting [21], besides the fact that asymptomatic cases can be only diagnosed during screening campaigns. For example, an ultrasound survey carried out by Caremani et al. [22] in 19 centres throughout Italy in 1988-90 found 424 cases diagnosed with CE by examining 333,144 patients, compared with 284 cases reported in the official data in the same period. Prevalence and incidence data in livestock are also incomplete $[17,19]$.

Difficulties in reporting of CE do not apply to Italy alone. Very heterogeneous national surveillance systems for CE exist in Europe [17]. However, comparison of notified cases with hospital records indicates a clear discrepancy, with consistent underestimation of the burden of CE $[17,18]$. For example, Pardo et al. reported a two- to fourfold lower number of notified cases compared with hospital records in certain regions of Spain from 1996 to 2003 [23].

Besides inaccurate reporting of the number of cases, other limitations of current reporting systems include ambiguity in discrimination between autochthonous 
World Health Organization Informal Working Group on Echinococcosis (WHO-IWGE) ultrasound classification of echinococcal cysts

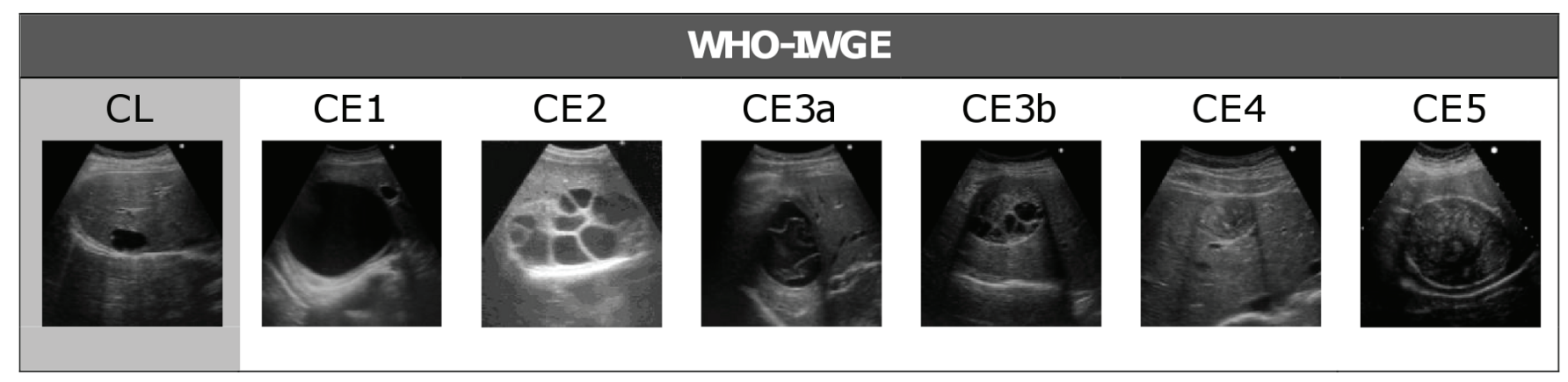

CL (cystic lesion), CE1 and CE2 (active cysts), CE33 and CE3b (transitional cysts), and CE4 and CE5 (inactive cysts). Source: [30].

and imported cases and between new and re-admitted cases, and lack of collection of important epidemiological data such as occupation and other risk factors. Given the chronicity and frequent relapses in CE $[24,25]$, patients often access different health centres over time, which could lead to potential duplication of data, which would also contribute to inaccurate statistics. In addition, aspects such as cyst stage and therapy outcome, which have a profound impact on the clinical management of patients and in turn on public health resource allocation, are generally not collected in notification forms.

\section{Setting up the Italian registry of cystic echinococcosis}

To start tackling these long-standing problems, the WHO Collaborating Centre for the Clinical Management of Cystic Echinococcosis, (University of Pavia, San Matteo Hospital Foundation, in Pavia) and the Istituto Superiore di Sanità (the Italian National Health Institute, ISS, in Rome) set up the Italian registry of cystic echinococcosis (Registro Italiano Echinococcosi Cistica, RIEC). The aims of the registry are to show the prevalence of CE in Italy, bring the importance of this infection to the attention of health authorities, encourage public health policies towards its control, and stimulate biological, epidemiological and clinical research on $\mathrm{CE}$.

\section{Organisation and management of the Italian registry}

The development of RIEC started in 2012 as part of a research project (IZS SA 07/10 RC) at the Sardinian Experimental Zooprophylactic Institute. The Project was managed by ISS and the Pavia WHO collaborating centre. The project group included a project manager $(\mathrm{PR})$, project coordinators (FT, EB), and an information technology developer (FG). After approval from the ethics committee of the ISS, RIEC was launched in October
2012, and is accessible through the ISS website [26]. The RIEC website [27] includes sections freely accessible to the public providing educational and scientific material, and a restricted area, accessible only to users authorised to enter data. The project group performs regular monitoring and evaluation of the database. Ownership of data from individual centres belongs to the individual centres themselves; however, upon permission, the project group analyses and publishes a cumulative summary of data on a regular basis.

\section{Data collection}

RIEC is a prospective, observational, multicentre registry of CE patients seen from January 2012 onward in Italian health centres that adhere voluntarily to the registration of their patients into RIEC. Clinicians of all disciplines in health centres potentially interested in joining RIEC are contacted by email and during scientific meetings. Clinicians are given a unique username and password to access the registry. Individual clinicians have access only to data of patients enrolled in their centres, and they can enrol and access data of CE patients previously recruited in another centre who subsequently moved and are then under their care.

The only inclusion criterion to enrol a new patient in the registry is that the patient has been diagnosed with probable or confirmed CE [11], whether hospitalised or in an outpatient setting and independent of country of birth and nationality. Upon first enrolment, a unique patient 'RIEC code' is generated automatically, avoiding duplication of records, should the patient present at another centre in the future, and facilitating data retrieval during follow-up. Following written informed consent concerning processing of sensitive data, other information can then be acquired (Box). These are entered using drop-down menus and multiple-choice options to facilitate data extraction and analysis, and can be updated following duplication and amendment. 


\section{FIGURE 2}

Preliminary data from patients with cystic echinococcosis enrolled in the Italian registry of cystic echinococcosis, Pavia, Italy (World Health Organization Collaborating Centre for Clinical Management of Cystic Echinococcosis), January 2012February $2014(\mathrm{n}=212)^{\circ}$

A Age at diagnosis and sex distribution

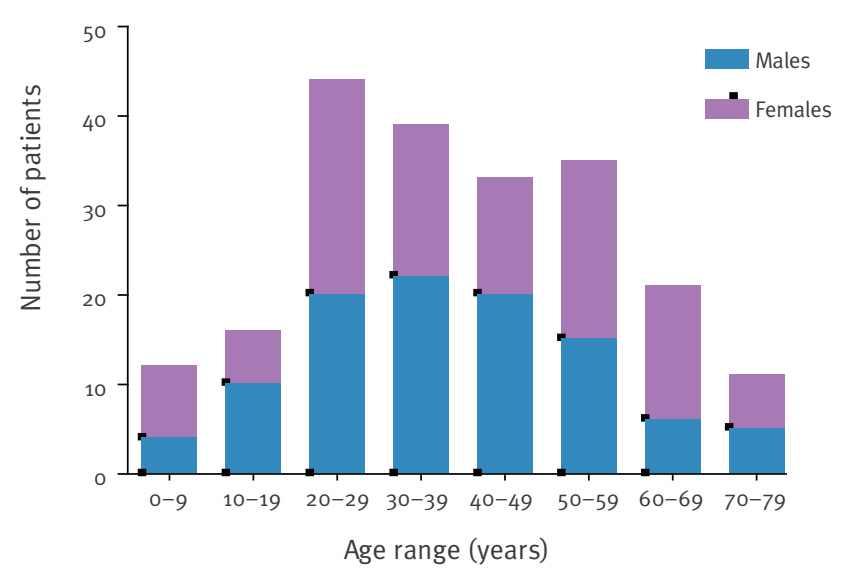

C Cysts per patient at first enrolment

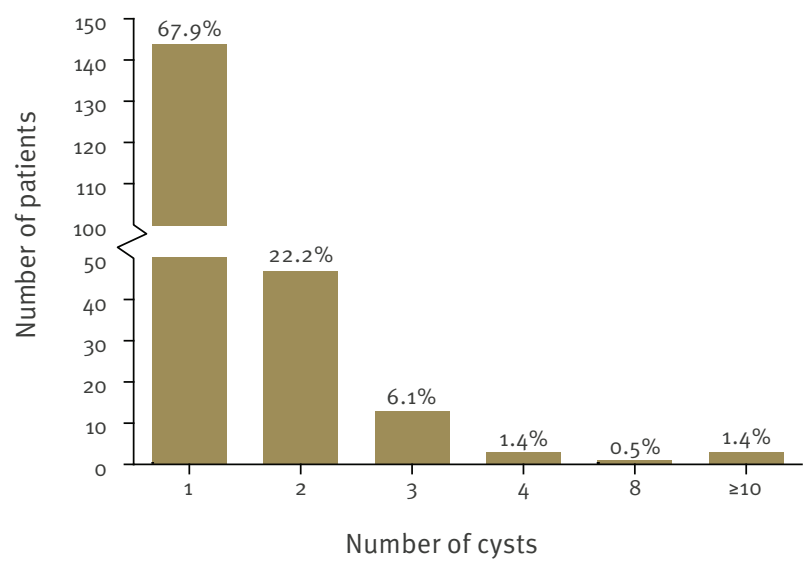

Data safety and confidentiality

RIEC is established within the secured ISS information technology network, in compliance with national and international regulations on the protection and use of personal data. Data are stored in a three-stage cluster database server implemented in a redundant array of independent disks and made available by an SQLrelational database management system server. The connection between authorised user and web application is made via an HTTP protocol. All registered data and logs are backed up at short regular intervals, and copied on a tape library stored in different fireproof locations. Upon patient registration, sensitive data are automatically anonymised with the assignment of a unique code, which is uncoupled from sensitive data to make them encrypted.
B Place of birth of patients

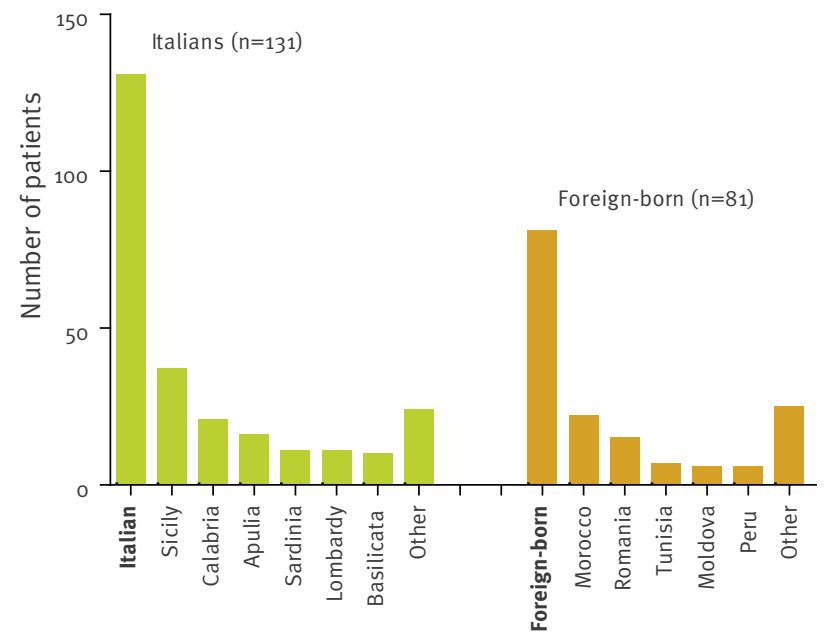

D Organ localisation of cysts $(n=361)$ and cyst stages within organs at first enrolment

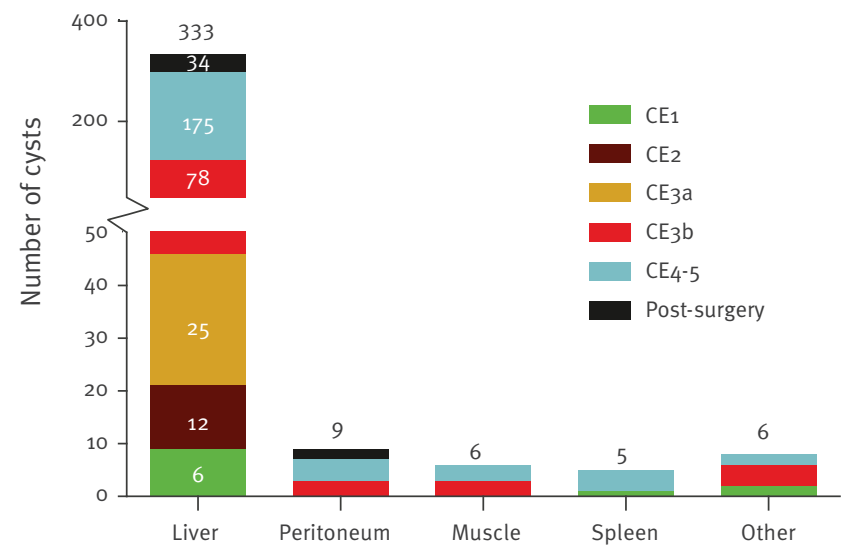

CE1 and CE2 (active cysts), CE3a and CE3b (transitional cysts), and CE4 and $\mathrm{CE}_{5}$ (inactive cysts).

\section{Preliminary findings}

By February 2014, 16 centres had adhered to the RIEC, with 346 patients enrolled in 11 centres. The remaining five centres had not yet had any enrolled patients. The majority of records (212 patients) were entered by the WHO collaborating centre in Pavia, showing a mean of 1.46 visits per patient (standard deviation (SD): 1.09; range: 1-4 visits/patient). Preliminary demonstrative results are derived from data from this centre.

A total of 110 male and 102 female CE patients were seen in this centre from January 2012 to February 2014 and were enrolled in the RIEC (Figure 2A). The mean age at diagnosis was 40 years (SD: 6.8 ; range: $5-77$ ) (Figure 2A). Of the 212 patients, $131(62 \%)$ were born in Italy, while 81 (38\%) were foreign-born (Figure 2B). 
Morocco and Romania were the countries of birth of the majority of foreign-born patients $(n=22$ and $n=$ 15 , respectively), while the majority of Italian patients were born in the southern regions of Sicily $(28 \%, n=$ 37 ) and Calabria ( $16 \%, n=21$ ) (Figure $2 B$ ). A total of 14 newly diagnosed cases were recorded in $2012(13 \%$ of 108 visits in 2012) and 21 in 2013 (21\% of 99 visits in 2013). During first enrolment, the majority $(70 \%$, $\mathrm{n}=148$ ) of patients had a single cyst (Figure $2 \mathrm{C}$ ), and 333 of 361 cysts (92\%) were hepatic (Figure 2D). Transitional (CE3a and $\mathrm{CE}_{3} \mathrm{~b}$ ) and inactive (CE4 and $\mathrm{CE}_{5}$ ) cysts stages were most commonly present at the time of enrolment (Figure 2D).

\section{Discussion}

The implementation of the RIEC responds to a longstanding need for a CE national registry with online data entry $[11,21]$, similar to the European registry for alveolar echinococcosis [28]. Its design allows the collection of both basic epidemiology and detailed longitudinal clinical data in a simple and unequivocal way. This will provide valuable information on the prevalence and parasitic pressure (linked to the number of small, active cysts) in patients in endemic areas. Moreover, it will offer the opportunity to study prospectively and systematically the stage-specific effectiveness of clinical management options and clinical manifestations, the rate of adverse reactions, relapse rate and costs of CE infection.

During January 2012 to February 2014, the Pavia centre entered the majority of the data, while the other centres entered only minimal demographic data. This was probably due to the fact that the Pavia centre is a pilot centre for RIEC implementation and is a WHO collaborating centre. Downloadable tools that facilitate data collection and entry, e.g. online tutorials and paperbased report forms for data collection during routine practice, are provided. However, more effort is needed from the project group to encourage data entry and highlight the advantages of using the RIEC, even for individual clinicians. Indeed, the RIEC allows systematic recording of data and of the clinical management of patients, even those followed in more than one centre. It also allows clinicians to retrieve and analyse the centre's cumulative clinical data for internal use and publications. Additionally, data collection and entry is not very time consuming: in Pavia, human resources allocated to this task were calculated as less than 0.02 full-time equivalents for 2013.

The fact that data entry relies on the clinician's goodwill and time suggests that the current figures are far from representing the real national situation. Only compulsory notification of CE would provide more reliable data for surveillance of the disease in humans. A centralised registry eliminates the need to merge regional data, avoids data duplication, captures both inpatient and outpatient data, and makes both clinical and epidemiological data accessible to clinicians, epidemiologists and health authorities. This is also of particular importance in light of increasing migration. It is crucial to inform control measures for what is essentially a preventable disease, and to allow for better resource allocation. The preliminary data from the RIEC presented here show that CE is still present in Italy and the cases largely outnumber the total of national cases reported by most European endemic countries [17], further stressing the need for a better reporting system for CE at the European level.

HERACLES (Human cystic Echinococcosis ReseArch in CentraL and Eastern Societies) is a four-year collaborative project launched in October 2013, funded by the European Union, under the Seventh Framework Programme (grant agreement number 602051). Within HERACLES, the RIEC has been be the template for the creation of the European registry of cystic echinococcosis, with initial inclusion of data from Bulgaria, Italy, Romania and Turkey, while health centres from other European countries expressed an interest in joining [29]. The European registry was launched in September 2014, is available in English and national languages, and is subject to constant monitoring and evaluation and template optimisation. This tool provides an efficient and disease-tailored template to governments, the European Commission and related European agencies to harmonise data collection, monitoring and reporting of CE.

\section{Acknowledgments}

The development of RIEC was funded through the Research Project IZSSA07/10RC to Dr Giovanna Masala (Sardinian Experimental Zooprophylactic Institute, Sassari, Italy), subcontracted to Enrico Brunetti. The amendment of RIEC and development of ERCE is funded through the European Union, under the Seventh Framework Programme (FP7, Theme Health.2013.2.3.4-1, grant agreement. no. 602051 to Adriano Casulli, Istituto Superiore di Sanità, Rome, Italy). We are thankful to Professor Giorgio Battelli (University of Bologna, Italy), and to Dr Simone Magnino (Experimental Zooprophylactic Institute of Lombardy and Emilia Romagna, Pavia, Italy) for their unflagging support. We are grateful to Professor Laura $\mathrm{H}$ Kramer, DVM, for reviewing the manuscript.

\section{Conflict of interest}

None declared.

\section{Authors' contributions}

FT conceived, designed and pilot tested RIEC and ERCE, contributed to data entry and RIEC and ERCE monitoring and evaluation, analysed the data and wrote the manuscript. PR designed and implemented the RIEC and ERCE website, contributed to RIEC and ERCE design and monitoring and evaluation, data analysis and manuscript writing. FG designed, pilot tested, and implemented RIEC and ERCE and contributed to data analysis and manuscript writing. MM, GJN and FR contributed to data entry and analysis. AC conceived and coordinated the implementation of ERCE and contributed to manuscript writing. EP contributed to the design of RIEC. EB conceived RIEC and ERCE, contributed to their design, and 
contributed to manuscript writing. All authors critically revised and approved the final version of the manuscript.

\section{References}

1. Craig PS, Budke CM, Schantz PM, Li T, Qiu J, Yang Y, et al. Human echinococcosis: a neglected disease? Trop Med Health. 2007;35(4):283-92. http://dx.doi.org/10.2149/tmh.35.283

2. Eckert J, Gemmell MA, Meslin F-X, Pawlowski ZS. WHO/OIE Manual on echinococcosis in humans and animals: a public health problem of global concern. Paris/Geneva: World Organization for Animal Health (Office International des Epizooties)/World Health Organization; 2001. Available from: http://whqlibdoc.who.int/publications/2001/929044522X.pdf

3. Food and Agriculture Organization of the United Nations (FAO)/ World Health Organization (WHO). Multicriteria-based ranking for risk management of food-borne parasites. Microbiological Risk Assessment Series No. 23. Rome. Rome/Geneva: FAO/ WHO; 2014. Available from: http://www.fao.org/3/a-i3649e.pdf

4. Budke CM, Deplazes P, Torgerson PR. Global socioeconomic impact of cystic echinococcosis. Emerg Infect Dis. 2006;12(2):296-303. http://dx.doi.org/10.3201/eid1202.050499 PMID:16494758

5. Brunetti E, Garcia HH, Junghanss T; International CE Workshop in Lima, Peru, 2009. Cystic echinococcosis: chronic, complex, and still neglected. PLoS Negl Trop Dis. 2011;5(7):e1146. http:// dx.doi.org/10.1371/journal.pntd.0001146 PMID:21814584

6. WHO Informal Working Group. International classification of ultrasound images in cystic echinococcosis for application in clinical and field epidemiological settings. Acta Trop. 2003;85(2):253-61. http://dx.doi.org/10.1016/S0001706X(02)00223-1 PMID:12606104

7. Frider B, Larrieu E, Odriozola M. Long-term outcome of asymptomatic liver hydatidosis. J Hepatol. 1999;30(2):22831. http://dx.doi.org/10.1016/S0168-8278(99)80066-X PMID:10068100

8. Macpherson CN, Milner R. Performance characteristics and quality control of community based ultrasound surveys for cystic and alveolar echinococcosis. Acta Trop. 2003;85(2):2039. http://dx.doi.org/10.1016/S0001-706X(02)00224-3 PMID:12606098

9. Hernández-González A, Muro A, Barrera I, Ramos G, Orduña A, Siles-Lucas M. Usefulness of four different Echinococcus granulosus recombinant antigens for serodiagnosis of unilocular hydatid disease (UHD) and postsurgical follow-up of patients treated for UHD. Clin Vaccine Immunol. 2008;15(1):14753. http://dx.doi.org/10.1128/CVI.00363-07 PMID:17989342

10. Gharbi HA, Hassine W, Brauner MW, Dupuch K. Ultrasound examination of the hydatic liver. Radiology. 1981;139(2):45963. http://dx.doi.org/10.1148/radiology.139.2.7220891 PMID:7220891

11. Brunetti E, Kern P, Vuitton DA; Writing Panel for the WHO-IWGE. Expert consensus for the diagnosis and treatment of cystic and alveolar echinococcosis in humans. Acta Trop. 2010;114(1):116. http://dx.doi.org/10.1016/j.actatropica.2009.11.001 PMID:19931502

12. Tamarozzi F, Nicoletti GJ, Neumayr A, Brunetti E. Acceptance of standardized ultrasound classification, use of albendazole, and long-term follow-up in clinical management of cystic echinococcosis: a systematic review. Curr Opin Infect Dis. 2014;27(5):425-31. http://dx.doi.org/10.1097/ QC0.0000000000000093 PMID:25101556

13. Profilassi dell' idatidosi. [Prophylaxis of hydatidosis]. Ordinanza del Ministero della Sanita' n. 21/04/1964 [Ordinance of the Italian Ministry of Health n. 21/04/1964]. Gazzetta Ufficiale della Repubblica Italiana [Official Journal of the Republic of Italy]. 8 May 1964, no. 112. Italian. Available from: http://www.gazzettaufficiale.it/do/gazzetta/foglio ordinario2/2/pdfPaginato?dataPubblicazioneGazzetta $=1964$ 0508\&numeroGazzetta=112\&tipoSerie $=$ FO\&tipoSupplemento $=\mathrm{GU} \&$ numeroSupplemento $=0$ \& numPagina $=5$ \&edizione $=0$ \& encozogiorni $=$

14. Aggiornamento dell'elenco delle malattie per le quali e' obbligatoria la denuncia ai sensi e per gli effetti dell'articolo 139 del testo unico approvato, con decreto del Presidente della Repubblica 30 giugno 1965, n. 1124 e successive modifiche e integrazioni. [Update of the list of diseases subject to compulsory notification according to article 139 of the unified document approved by decree of the President of the Republic 30 June 1965, n. 1124 and subsequent amendments and integrations]. Decreto del Ministero del Lavoro n. 11/12/2009. [Decree of the Italian Ministry of Work n. 11/12/2009]. Gazzetta Ufficiale della Repubblica Italiana. [Official Journal of the Republic of Italy]. 19 Mar 2010, no. 65. Italian. Available from:http://www.gazzettaufficiale.it/
atto/serie_generale/caricaDettaglioAtto/originario?atto. dataPubblicazioneGazzetta=2010-03-19\&atto.codiceRedaziona $l e=10 A 03140 \&$ normativi $=$ false\&tipoVigenza $=$ originario\&tipoS erie $=$ serie_generale $\&$ currentPage $=1$

15. Attuazione della direttiva $2003 / 99 /$ CE sulle misure di sorveglianza delle zoonosi e degli agenti zoonotici. [Implementation of Directive 2003/99/ EC on the monitoring of zoonoses and zoonotic agents]. Gazzetta Ufficiale della Repubblica Italiana. [Official Journal of the Republic of Italy]. 24 May 2006, no. 119. Italian. Available from: http://www. gazzettaufficiale.it/eli/id/2006/05/24/006G0210/sg

16. Sistema informativo delle malattie infettive e diffusive. [Notification system of infectious and communicable diseases] Decreto del Ministero della Sanita' n. 15/12/1990. [Decree of the Italian Ministry of Health n. 15/12/199o]. Gazzetta Ufficiale della Repubblica Italiana. [Official Journal of the Republic of Italy]. 8 Jan 1991, no. 6. Italian. Available from: http://www. gazzettaufficiale.it/atto/serie_generale/caricaDettaglioAtto/ originario?atto.dataPubblicazioneGazzetta=1991-01-08\&atto. codiceRedazionale $=091 \mathrm{~A} 0055$

17. European Centre for Disease Prevention and Control (ECDC). Annual epidemiological report 2013. Reporting on 2011 surveillance data and 2012 epidemic intelligence data. Stockholm: ECDC; 2013. Available from: http://ecdc.europa. eu/en/publications/Publications/Annual-EpidemiologicalReport-2013.pdf

18. Rojo-Vazquez FA, Pardo-Lledias I, Francos-Von Hunefeld M, Cordero-Sanchez M, Alamo-Sanz R, Hernandez-Gonzalez $A$, et al. Cystic echinococcosis in Spain: current situation and relevance for other endemic areas in Europe. PLoS Negl Trop Dis. 2011;5(1):e893. http://dx.doi.org/10.1371/journal. pntd.0000893 PMID:21283615

19. European Food Safety Authority, European Centre for Disease Prevention and Control. The European Union summary report on trends and sources of zoonoses, zoonotic agents and food-borne outbreaks in 2011. EFSA Journal. 2013;11(4):3129. doi:10.2903/j.efsa.2013.3129

20. Brundu D, Piseddu T, Stegel G, Masu G, Ledda S, Masala G. Retrospective study of human cystic echinococcosis in Italy based on the analysis of hospital discharge records between 2001 and 2012. Acta Trop. 2014;140:91-6. http://dx.doi. org/10.1016/j.actatropica.2014.08.011 PMID:25149351

21. Petrone L, Cuzzi G, Colace L, Ettorre GM Busi-Rizzi E, Schininà $V$, et al. Cystic echinococcosis in a single tertiary care center in Rome, Italy. Biomed Res Int. 2013;2013:978146. doi: $10.1155 / 2013 / 978146$

22. Caremani M, Maestrini R, Occhini U, Sassoli S, Accorsi A, Giorgio A, et al. Echographic epidemiology of cystic hydatid disease in Italy. Eur J Epidemiol. 1993;9(4):401-4. http://dx.doi. org/10.1007/BFo0157397 PMID:8243595

23. Pardo J, Muro A, Galindo I, Cordero M, Carpio A, SilesLucas M. [Hydatidosis in the province of Salamanca (Spain): should we let down our guard?]. Enferm Infecc Microbiol Clin. 2005;23(5):266-9. Spanish. http://dx.doi. org/10.1157/13074966 PMID:15899176

24. Rinaldi F, De Silvestri A, Tamarozzi F, Cattaneo F, Lissandrin R, Brunetti E. Medical treatment versus "Watch and Wait" in the clinical management of CE3b echinococcal cysts of the liver. BMC Infect Dis. 2014;14:492.

25. Stojkovic M, Zwahlen M, Teggi A, Vutova K, Cretu CM, Virdone $\mathrm{R}$, et al. Treatment response of cystic echinococcosis to benzimidazoles: a systematic review. PLoS Negl Trop Dis. 2009;3(9):e524.

26. Istituto Superiore di Sanità (ISS). Istituto Superiore di Sanità. Rome: ISS. [Accessed 5 May 2015]. Italian. Available from: http://www.iss.it

27. Registro Italiano dell'Echinococcosi Cistica. [Italian register of cystic echinococcosis.] [Accessed 5 May 2015]. Italian. Available from: http://www.iss.it/riec

28. Kern P, Bardonnet K, Renner E, Auer H, Pawlowski Z, Ammann RW, et al.; European echinococcosis registry: human alveolar echinococcosis, Europe, 1982-2000. Emerg Infect Dis. 2003;9(3):343-9. http://dx.doi.org/10.3201/eido903.020341 PMID:12643830

29. HERACLES. ERCE. European Register of Cystic Echinococcosis. Rome: Istituto Superiore di Sanità. [Accessed 5 May 2015] Available from: http://www.heracles-fp7.eu/erce.html

30. Piccoli L, Tamarozzi F, Cattaneo F, Mariconti M, Filice C, Bruno A, et al. WHO-IWGE ultrasound classification of echinococcal cysts. Figure_1.tif. Long-term sonographic and serological follow-up of inactive echinococcal cysts of the liver: hints for a "watch-and-wait"approach. PLoS Negl Trop Dis. 2014;8(8):e3057. 10.1371/journal.pntd.0003057.g001 